\title{
Stability of Water Lubricated Flow of Yield Stress Fluid in Sloping Pipe
}

\author{
A. Ahmad ${ }^{1, a}$, B. Nsom ${ }^{1}$, and J.P. Decruppe ${ }^{2}$ \\ ${ }^{1}$ Université de Bretagne Occidentale, LBMS EA 4325. Rue de Kergoat. 29231 BREST, France \\ ${ }^{2}$ Université Paul Verlaine, ICPM, 1, Bld Dominique ARAGO. F-57078 METZ Cedex, France
}

\begin{abstract}
To facilitate the transport of viscous crudes in a pipe, an immiscible lubricating liquid, usually water, is added. In such configuration, the water migrates into the regions of high shear at the pipe wall where it lubricates the flow. The pumping pressures being balanced by wall shear stresses in the water, the flow therefore requires pressures comparable to pumping water alone, at the same total throughput [1]. So significant savings in pumping power can be derived from this process provided that it is well monitored. Indeed, instabilities usually take place at the oil/water interface and they constitute an important source of energy dissipation. Precisely, a core annular flow is known to undergo a long-wave instability of capillary type, modified by shear occuring at low Reynolds. Above a given critical Reynolds number, the flow is unstable to shorter waves which leads to an emulsification system of water droplets in oil. In present work, an experimental study of the stability of sloping plane Poiseuille flow of well characterized viscoplastic mineral oils lubricated by water was performed. The investigation was carried out by means of image analysis based on spatiotemporal diagrams (STD). Notably indicated are the effects of bed slope, flow rates ratio and oil rheology on flow stability.
\end{abstract}

\section{Introduction}

The flow of a water/oil mixture in a pipe has two main characteristics. Firstly, the fluids can adopt different spatial arrangements called flow regimes and secondly, the presence of a water layer at channel wall significantly reduces the global pressure drop. Among the various arrangements which occur in such flowing binary fluids, the concentric oil in water, also called core-annular flow (CAF) is the desired one as it provides the optimal energy efficiency. A thorough state-of-the-art on annular flows and their industrial applications is provided by Joseph and Renardy [2].

The lubricating-film model assumes that waves on the oil/water interface play a major role in the momentum balance of the flow in the water. In this model, it is then possible to estimate the pressure gradient for core-annular flow in a horizontal pipeline, provided that the wavelength, amplitude and shape of these waves are known [3]. Now, focusing on the special case of co-courant flow of two liquids in plane Poiseuille configuration, literature provides less papers than for the cylindrical case. Charles \& Lilleleht [4] determined the laminar-turbulent transitions for both phases experimentally.

a e-mail : ahmad.ahmad@univ-brest.fr

This is an Open Access article distributed under the terms of the Creative Commons Attribution-Noncommercial License 3.0, which permits unrestricted use, distribution, and reproduction in any noncommercial medium, provided the original work is properly cited. 
Notably, the transition to turbulence in the water phase occurred at a higher Reynolds number in presence of a laminar oil layer provided the input water-to-oil ratio was relatively high, while the transition in the oil phase took place at a lower Reynolds number in the presence of a turbulent water layer. Furthermore, the appearance of first interfacial waves coincided with the transition to turbulence in the water phase. Yih [5] performed a linear study of the stability of two superposed fluids in plane Poiseuille flow subjected to two-dimensional long-wavelength disturbances. He derived an expression for the complex wave speed and showed that viscosity stratification can give rise to an interfacial mode that is unstable for arbitrarily small Reynolds number. In their experimental investigation of stability of flow of superposed fluids in a rectangular channel, Kao \& Park [6] observed that only the disturbances in water grew in the beginning stages of transition to turbulence. They provided mean flow profiles, the amplitude distribution of disturbances in water, the amplification rate, wave speed and wavenumbers. They also showed that there was a critical Reynolds number beyond which the flow was unstable to a shear mode of Tollmien-Schlichting type that gave rise to interfacial waves. Yiantsios \& Higgins [3] determined numerically the conditions for the the growth of an interfacial wave in plane Poiseuille flow of two superposed fluids of different viscosity. Their analysis extends Yih's results [5] for small wavenumbers to large wavenumbers, accounts for differences in density and thickness ratios, as well as the effects of interfacial tension and gravity.

In this paper, the stability of CAF in sloping plane Poiseuille flow is investigated experimentally. The experimental set-up and the procedure are presented in the second section, while the experimental results are provided in the third section. Notably indicated are the effects of bed slope, flow rates ratio and oil rheology on flow stability.

\section{Experimental set-up and procedure}

An experimental set-up with a $13 \mathrm{~m}$ long transparent channel and $4 \mathrm{x} 4 \mathrm{~cm}$ cross section as principal organ was designed and built in the laboratory. The device head consists of two distinct circuits, one for the water and the other one for the oil (fig. 1). The flow was maintained by constanthead tanks for both water and oil and was re-circulated by pumps. Each tank was provided with an overflow device to maintain a constant head.

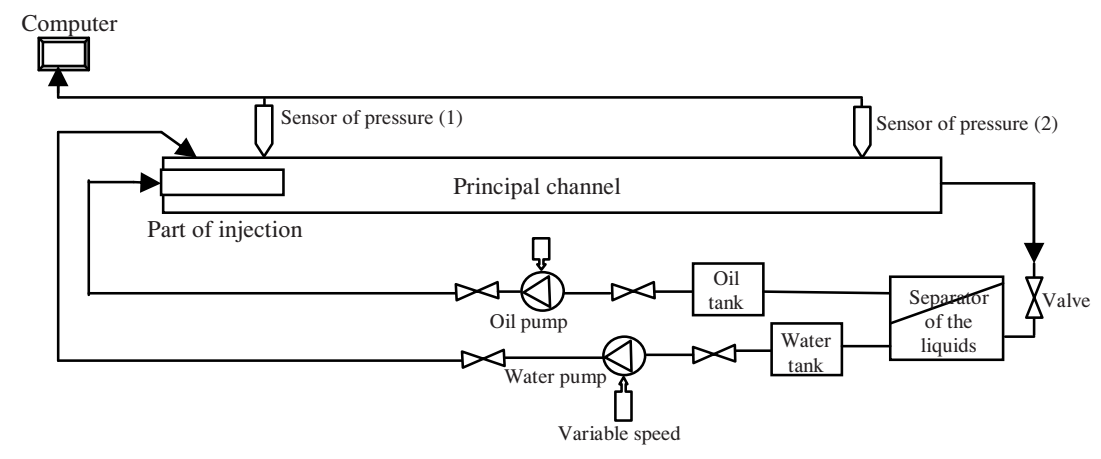

Fig. 1. Experimental set-up

Each tank was provided with an overflow device to maintain a constant head. The flow was allowed to mix during discharge from the channel and the mixture was separated in a separator tank (CSD-B Séparateur hydrocarbures Acier $60 \times 60 \times 80 \mathrm{~cm}$ ), able to treat 11/s. The head in the separator tank was kept constant by the overflow of oil by using an interface sensing probe. The flow rate and the temperature were kept steady. The set-up was validated by comparing the measured pressure drop 
with the theoretical value in both horizontal and inclined plane Poiseuille flow of Newtonian fluid. It should be stressed that both fluids, the oil and the water are used in closed circuit for obvious reasons of water economy. Meanwhile, to ensure the quality of the fluids used, regularly during the experiments, samples are picked in each reservoir and characterized. Furthermore, the literature on water-lubricated transport of heavy viscous oils is generally brought out in horizontal and vertical pipes and Newtonian oils [2]. In this work, the device frame was equipped with a system of screws which can give an assigned slope to the pipe, while the working fluids were ordinary tap water and three types of Blasia P mineral oil supplied by Agip Oil Company. The rheological characterization of the oils was achieved with the help of a Carrimed CSL 100 rheometer. A Bingham behaviour was obtained as shown on rheograms presented in figures 2 and 3 for the Blasia P 1000 oil.

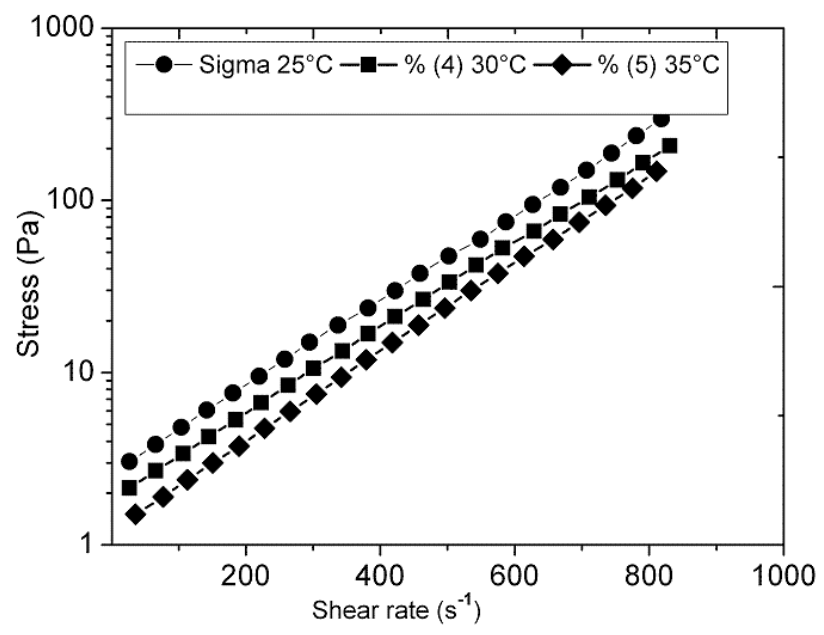

Fig. 2. Shear stress-shearing rate rheogram of Blasia P 1000 oil

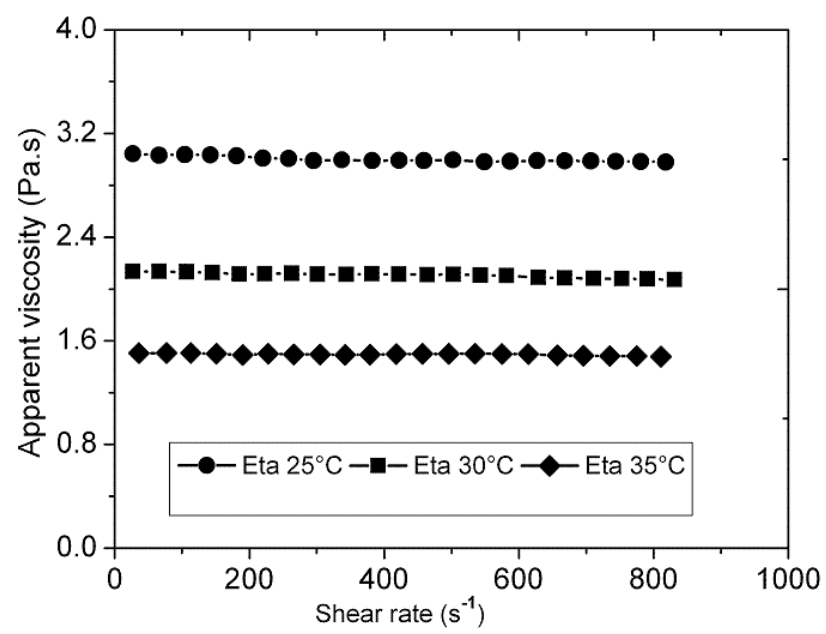

Fig. 3. Viscosity-shearing rate rheogram of Blasia P 1000 oil 
Moreover, the interfacial tension was measured using a 2D Dunouy numerical and automatic tensiometer. The pressure drop was characterized using electronic probes (Keller) of $0.01 \%$ accuracy, while the flow stability was investigated by means of image analysis. The flow images were recorded using a fast camera (Photron) of 250 images/sec and a resolution of $1024 \times 1024$ pixels. Spatio-temporal diagrams (STD) of the flow were then extracted from these images to track the interface shape. Programs were developed on the Matlab platform to make that procedure automatic.

\section{Flow stability}

Flow regimes maps of the patterns occurring in the flow against the respective flow rates were performed for assigned channel slope. The stability study was carried out on CAF regime (fig. 4) by means of image analysis for a target of values for water flow rate $\left(\mathrm{Q}_{\text {water }}\right)$, oil flow rate $\left(\mathrm{Q}_{\text {oil }}\right)$ and channel slope (alpha) for each oil.

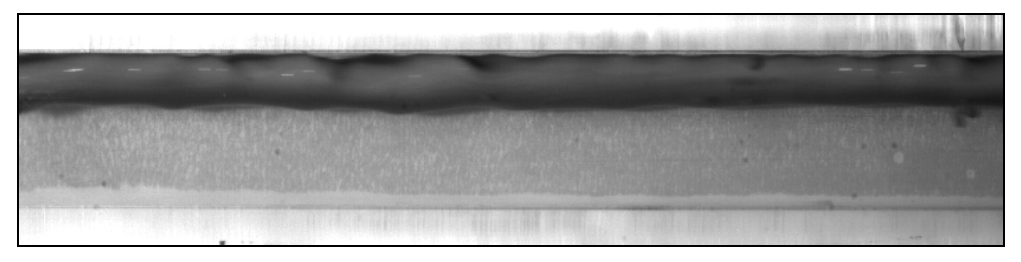

Fig. 4. Typical pattern of core-annular flow pattern observed

From the images taken with the Photron camera described in previous section, we derived the horizontal STD, i.e. the disturbance evolution in the $(\mathrm{x}, \mathrm{t})$ plane, as shown in fig.5. Wavelength, period and wave velocity can

be measured on this diagram.

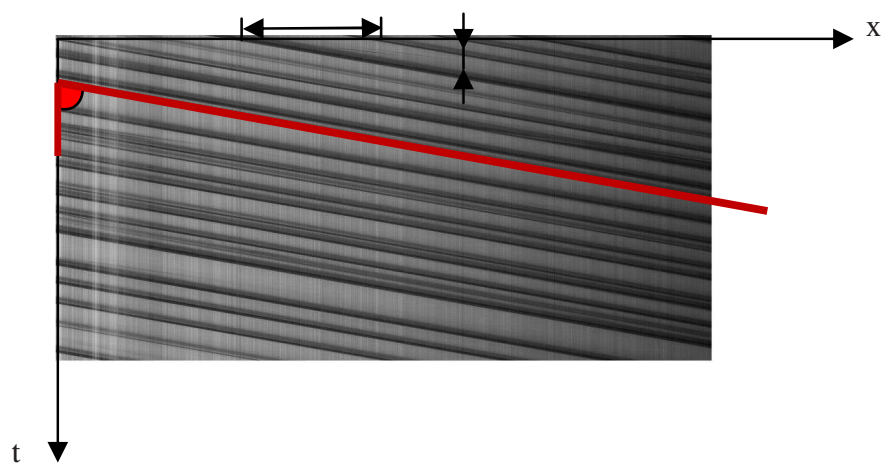

Fig. 5. Horizontal spatiotemporal diagram

Also, the vertical STD, i.e. the disturbance evolution in the $(y, t)$ plane, as shown in fig.6 was derived. Time variation of amplitude at given $\mathrm{x}$ can be measured on that other diagram. Applying then a FFT on these STD we obtained the signal frequency and spectral density as shown in fig.7. 


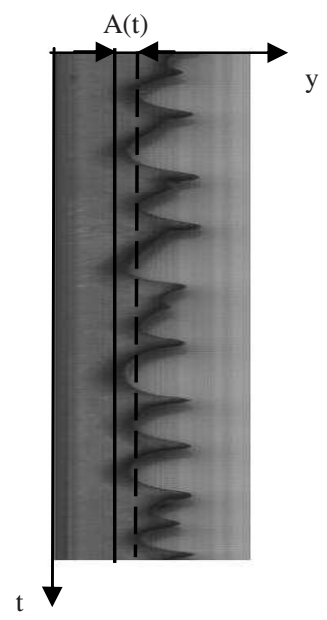

Fig. 6. Vertical spatiotemporal diagram
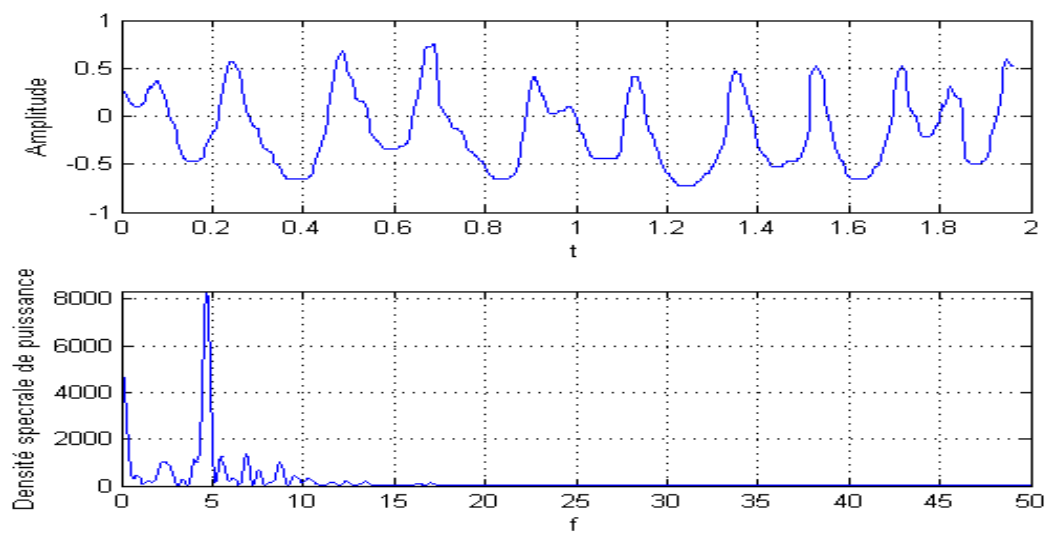

Fig. 7. Signal analysis of amplitude

The following results were obtained with the Blasia P 1000 oil. The amplitude increases with $\mathrm{x}$, as shown in fig.8 while it decreases with increasing $\mathrm{Q}_{\text {water }}$ or $\mathrm{Q}_{\text {oil }}$ as shown in fig.9 or channel slope

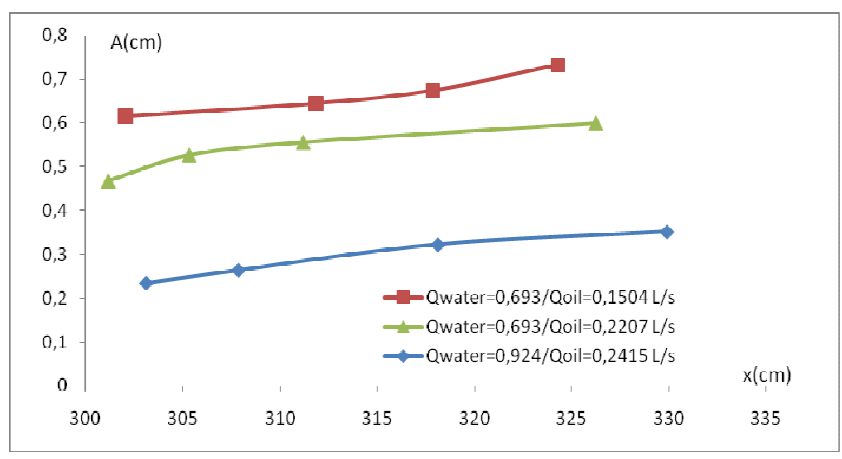

Fig. 8. Variation of amplitude along channel length 


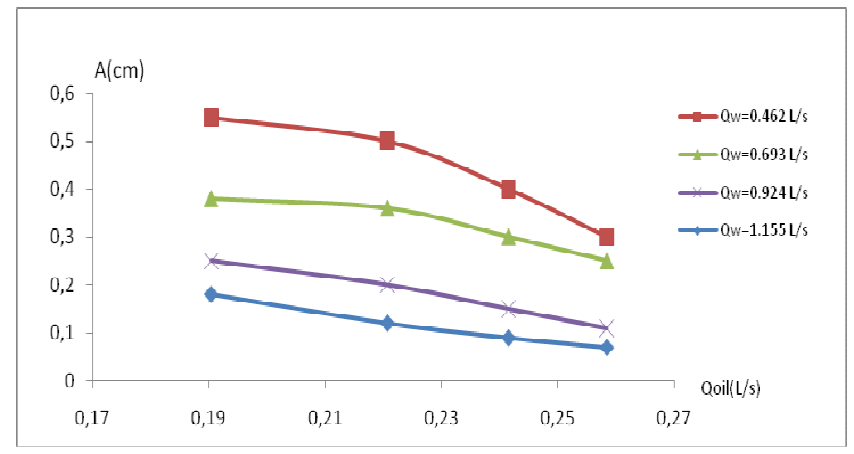

Fig. 9. Variation of amplitude with water flow rate $\mathrm{Q}_{\mathrm{w}}$ and oil flow rate $\mathrm{Q}_{\text {oil }}$

as shown in fig.10. The wave velocity increases with increasing $\mathrm{Q}_{\text {water }}$ or $\mathrm{Q}_{\text {oil }}$ as shown in fig.11 or channel slope as shown in fig. 12 .

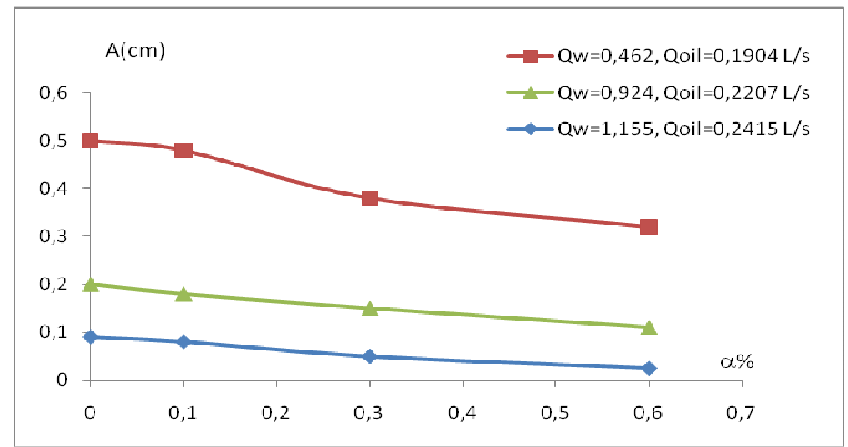

Fig. 10. Variation of amplitude with channel slope

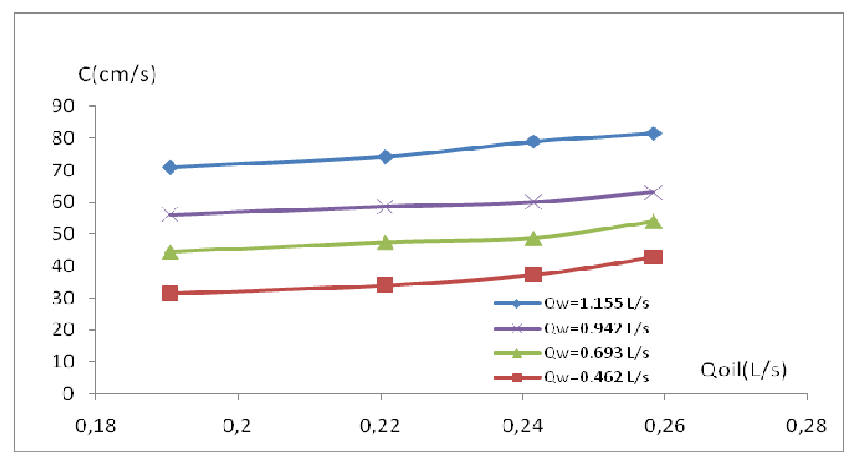

Fig. 11. Variation of wave velocity with water flow rate $Q_{w}$ and oil flow rate $Q_{\text {oil }}$ 


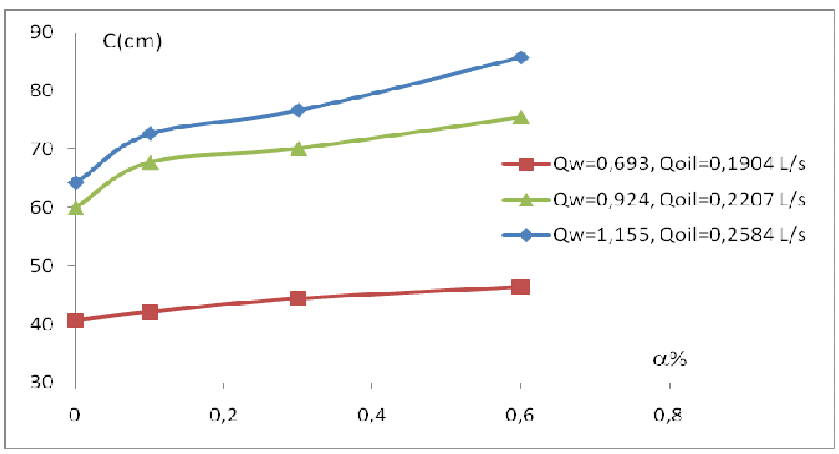

Fig. 12. Variation of wave velocity with channel slope

The frequency decreases with increasing $\mathrm{Q}_{\text {water }}$ or $\mathrm{Q}_{\text {oil }}$ as shown in fig.13, or channel slope as shown in fig.14, while the wavelength increases with increasing $Q_{\text {water }}$ or $Q_{\text {oil }}$ as shown in fig.15 or channel slope as shown in fig. 16.

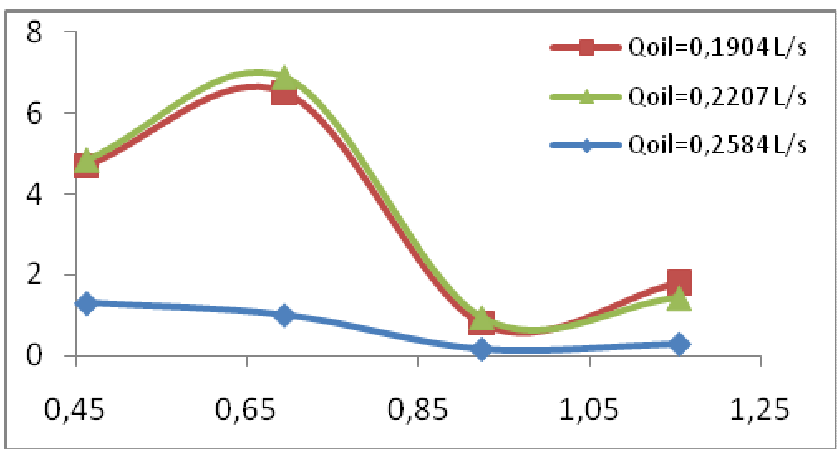

Fig. 13. Variation of frequency with water flow rate $Q_{w}$ and oil flow rate $Q_{o i l}$

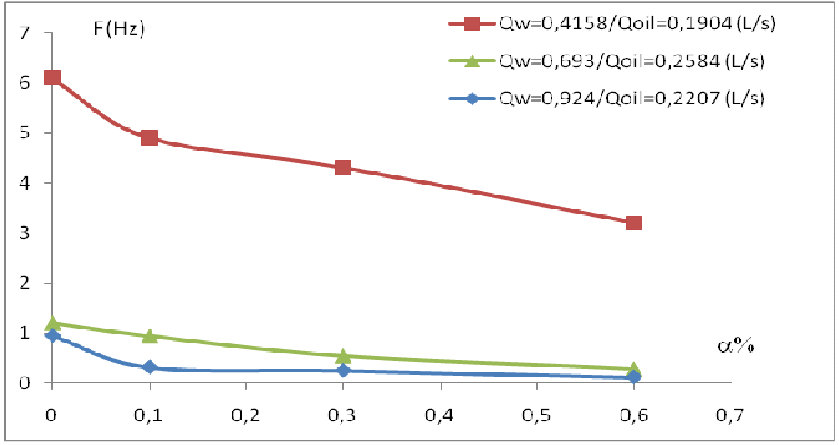

Fig. 14. Variation of frequency with channel slope 


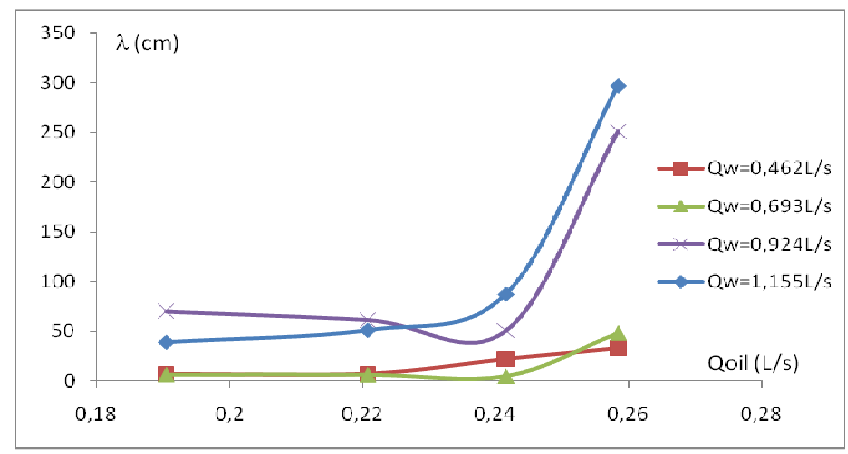

Fig. 15. Variation of wavelength with water flow rate $\mathrm{Q}_{\mathrm{w}}$ and oil flow rate $\mathrm{Q}_{\text {oil }}$

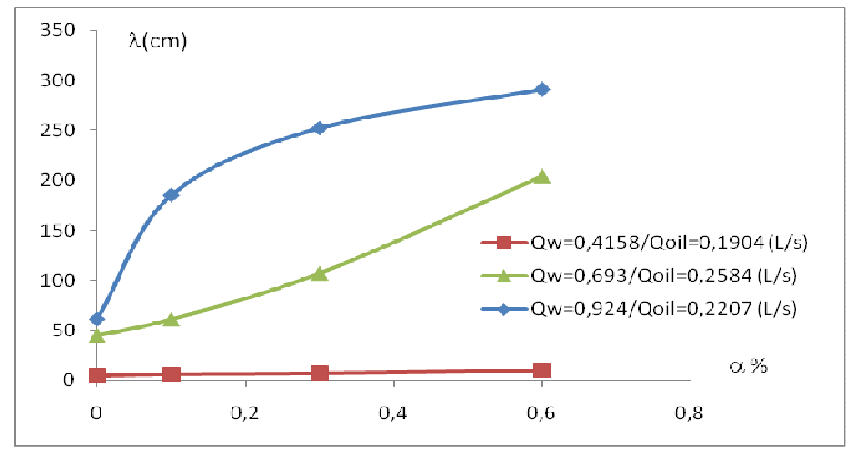

Fig. 16. Variation of wavelength with channel slope

Assuming that the flow becomes more stable with decreasing amplitude or frequency and increasing wavelength, these experimental results show that increasing $\mathrm{Q}_{\text {water }}$ or Qoil or channel slope makes the flow more stable. Analogous results were obtained for the 2 other oils, and for given $\mathrm{Q}_{\text {water }}$ or $\mathrm{Q}_{\text {oil }}$ or channel slope, a more stable flow was obtained with a more viscous oil. It means that for assigned $\mathrm{Q}_{\text {water }}, \mathrm{Q}_{\text {oil }}$ and channel slope, the flow was more stable with the Blasia P 1000 oil and less stable with the Blasia P 480 oil.

All these results can be useful for the design and operation of crude-oil flow lines and directional wells.

\section{References}

1. P.A.M. Boomkamp, R.H.M. Miesen, Phys. Fluids A 4, 1627 (2004)

2. D.D. Joseph, Renardy, Y.Y., Fundamentals of Two-Fluid Dynamics (Springer, New York, 1993)

3. S.G. Yiantsios, B.G. Higgins, Phys. Fluids 31, 3225 (1988)

4. M.E. Charles, L.U. Lilleleht, J. Fluid Mech. 22, 217 (1965)

5. C.S. Yih, J. Fluid Mech. 27, 337 (1967)

6. T.M. Kao, C. Park, J. Fluid Mech. 52, 401 (1972) 